\title{
Demographic noise and cost of greenbeard can facilitate greenbeard cooperation
}

\author{
Jaideep Joshi' ${ }^{1,2}$ iD and Vishwesha Guttal ${ }^{1}$ iD \\ ${ }^{1}$ Centre for Ecological Sciences, Indian Institute of Science, Bengaluru 560012, India \\ ${ }^{2}$ E-mail: jaideepjoshi@iisc.ac.in
}

Received April 16, 2018

Accepted September 22, 2018

\begin{abstract}
Cooperation among organisms, where cooperators suffer a personal cost to benefit others, is ubiquitous in nature. Greenbeard is a key mechanism for the evolution of cooperation, where a single gene or a set of linked genes codes for both cooperation and a phenotypic tag (metaphorically called "green beard"). Greenbeard cooperation is typically thought to decline over time since defectors can also evolve the tag. However, models of tag-based cooperation typically ignore two key realistic features: populations are finite, and that phenotypic tags can be costly. We develop an analytical model for coevolutionary dynamics of two evolvable traits in finite populations with mutations: costly cooperation and a costly tag. We show that an interplay of demographic noise and cost of the tag can induce coevolutionary cycling, where the evolving population does not reach a steady state but spontaneously switches between cooperative tag-carrying and noncooperative tagless states. Such dynamics allows the tag to repeatedly reappear even after it is invaded by defectors. Thus, we highlight the surprising possibility that the cost of the tag, together with demographic noise, can facilitate the evolution of greenbeard cooperation. We discuss implications of these findings in the context of the evolution of quorum sensing and multicellularity.
\end{abstract}

KEY WORDS: Coevolution, demographic noise, evolutionary dynamics, greenbeard, sociality.

Cooperation is an act where an individual confers a benefit to a recipient at a personal cost. A well-mixed population of cooperators can be invaded by defectors, who receive the benefits from cooperators but do not contribute any benefits in return nor suffer any cost. Cooperators can resist invasion by defectors if they assort positively, that is when benefits of cooperation are directed preferentially toward other cooperators (Hamilton 1975; Pepper 2000; Fletcher and Doebeli 2009). One of the ways in which positive assortment can occur is if a single gene (or a set of linked genes) simultaneously codes for cooperation and an assortment mechanism (Gardner and West 2010). Such genes are called "greenbeards" (Hamilton 1964; Dawkins 1976, 1982). A couple of greenbeard genes have indeed been discovered: for example, in microbes, a single gene may code for cellular adhesion (which sorts adhesive cells from nonadhesive cells) and cooperation in the form of public-goods production (Queller et al. 2003; Smukalla et al. 2008). Greenbeard effects are also observed for behaviors other than cooperation: in the red fire ant, linked genes code for the greenbeard (in the form of a specific odor cue) and spite (in the form of workers killing queens who do not bear the same genotype; see Keller and Ross (1998)). However, such examples of a single-gene or linked-genes coding for two different traits are only a handful. Therefore, greenbeard mediated behaviors are considered to be rare in nature (Dawkins 1976).

Yet, species across taxa are known to positively assort using greenbeard mechanisms. These are typically in the form of phenotypic or behavioral markers, or "tags". For example, a number of microbial species secrete adhesive molecules that passively segregate adhesive cells from nonadhesive cells. Cells in the adhesive clusters cooperate to produce public goods (Cooksey and Wigglesworth 1995; Hall-Stoodley et al. 2004; Drescher et al. 2014). Quorum sensing bacteria use a chemical signal to assess the density of cells in their neighborhood, and produce public goods only if the signal level is above a threshold (Waters and Bassler 2005; Czárán and Hoekstra 2009). Even behaviors such as roosting at a particular site or being active only at a specific time of the day (Caccamise and Morrison 1986) have the potential to segregate individuals and facilitate assortment. In fact, 
theory predicts that such phenotypic or behavioral sorting can arise via simple mechanisms such as differential speed or cohesion among individuals (Couzin et al. 2002; Guttal and Couzin 2010; Garcia and De Monte 2013; Garcia et al. 2014; Joshi et al. 2017). Assortment of such phenotypes (tags) would create a corresponding assortment of cooperators so long as only cooperators carry these traits. However, theoretical studies show that such an association between cooperative behavior and the tag is prone to decay over time as defectors evolve the tag (Roberts and Sherratt 2002). Therefore, despite the prevalence of various phenotypic sorting mechanisms, tag-based cooperation is considered to be theoretically less viable (Lehmann and Keller 2006).

A number of theoretical studies have investigated mechanisms for tag-based cooperation (Riolo et al. 2001; Roberts and Sherratt 2002; Hammond and Axelrod 2006; Traulsen and Nowak 2007; Zhang and Chen 2016). Riolo et al. (2001) proposed that tag-based cooperation can persist if all individuals conditionally cooperate with phenotypically similar individuals, and defect otherwise. However, unconditional defectors were shown to readily invade such cooperators (Roberts and Sherratt 2002). Traulsen and Schuster (2003) revisited this problem and showed that random changes between conditional and unconditional cooperators create fluctuations in their proportions, and allow cooperators and tag-carriers to sustain. However, they did not consider unconditional defectors in their model. Therefore, conditions that prevent an invasion by unconditional defectors remain unclear. Subsequently, researchers showed that if multiple phenotypic markers are possible, cooperators continuously discover newer markers as defectors catch up and begin to invade. This mechanism, called "beard-chromodynamics" has recently gained popularity (Jansen and van Baalen 2006; Traulsen and Nowak 2007; Antal et al. 2009). However, most studies on tag-based cooperation ignore some key features of real populations. First, real populations are finite. Genetic drift in such populations can lead to counterintuitive evolutionary dynamics, including, for example, reversal of the direction of selection (Kogan et al. 2014). Second, tags often are costly. For example, production of signaling or adhesive molecules in bacteria incurs metabolic costs (Nadell et al. 2009), and individuals carrying a conspicuous phenotype could be at an increased risk from predators. Third, most such studies assume that all individuals possess one of many tags, and that individuals interact with others possessing a similar tag. Such a model formulation, however, may not adequately represent individuals with cellular adhesion or signaling as tag-carriers; this is because, as shown by spatially explicit simulations, whereas adhesive molecules do interact to form groups, nonadhesive/nonsignaling (tagless) individuals are likely to remain solitary and rarely interact with others (Garcia et al. 2014). In our work, we aim to fill in these gaps by considering the role of cost of tag in finite populations, when only one tag is present, on the evolution of greenbeard cooperation.

Here, we develop an analytical model for greenbeard cooperation in finite populations where each individual has two evolvable traits: cooperation (cooperator or defector) and a tag (tag-carrier or tagless). Starting with microscopic (individual level) and stochastic processes of death, reproduction, and mutation, we use the continuous time chemical Langevin equation (Gillespie, 2000) to arrive at the macroscopic (population level) evolutionary dynamics. We show that mutations, demographic noise arising from finite population size and cost of the tag together can lead to a spontaneous emergence of an association between cooperation and a phenotypic sorting mechanism, a hallmark of greenbeard cooperation. We find this to be true in both prisoner's dilemma and public goods games. We then discuss our study in the context of the evolution of grouping behaviors, microbial adhesion, multicellularity, and quorum sensing.

\section{Analytical Selection-Mutation-Drift Model}

We consider a population of $N$ individuals, where each individual has two independent (unlinked) binary traits. The first is a binary behavioral trait $\omega_{c i}$, which determines whether an individual cooperates $\left(\omega_{c i}=1\right)$ or defects $\left(\omega_{c i}=0\right)$. Cooperation and defection are assumed to be unconditional, that is cooperators always cooperate and defectors always defect. The second is a binary phenotypic trait $\omega_{t i}$, which determines whether or not the individual carries a certain phenotypic tag. Thus, there are four types of individuals (or four "species") in the population (Fig. 1): tagless cooperators $\left(S_{1}\right)$, tag-carrying cooperators $\left(S_{2}\right)$, tagless defectors $\left(S_{3}\right)$, and tag-carrying defectors $\left(S_{4}\right)$. Let the proportion of species $S_{i}$ be $x_{i}$. The set $\boldsymbol{x}=\left\{x_{1}, x_{2}, x_{3}, x_{4}\right\}$ describes the state of the system at any given time. We keep the population size constant. Therefore, $\sum x_{i}=1$, and any three variables are sufficient to completely describe the state of the population. The changes in the state of the system occur in continuous time by mutation and selection via 12 channels as shown in Fig. 1 and Table S1. Due to finite population size, the system also experiences demographic stochasticity or genetic drift. We use the framework of the chemical Langevin equation (Gillespie 2000) to describe the dynamics of the state variables $x_{i}$, as described below.

\section{MUTATION}

The microscopic (or individual level) process for mutations is such that each trait mutates at a rate $\mu$. We assume that the mutation rate is very small, and thus, neglect simultaneous mutations in both traits that would occur at a rate $\mu^{2}$. Mutations change the state of the system. For example, a mutation of a tagless cooperator 


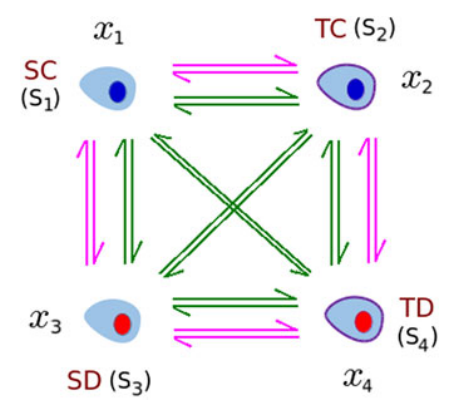

Figure 1. Schematic of our model with individual types and transition channels. The system contains four types of individuals ("species") $S_{1}-S_{4}$. In a given individual, depicted here as a cell, thick outline indicates the presence of the tag, and the color of the nucleus depicts cooperation (red is defector and blue is cooperator). The proportion of species $S_{i}$ is $x_{i}$. S1 represents solitary cooperator (SC), S2 is tagged cooperator (TC), S3 is solitary defector (SD) and S4 is tagged defector (TD). The six magenta arrows represent mutations and eight olive arrows represent death-birth (selection). There are a total of 12 channels, with four channels allowing selection alone (since we neglect double-mutations that would be required in these channels), and eight channels allowing both selection and mutation. In such a population, for example, the propensity for an SC to convert to TC is given by equation (9), which shows the role of both mutation and selection, as well as the density of other types of individuals. See equation (10) for rates of all such transitions.

$\left(S_{1}\right)$ into a tag-carrying cooperator $\left(S_{2}\right)$ will cause the frequencies to change as follows: $\left(x_{1}, x_{2}, x_{3}, x_{4}\right) \rightarrow\left(x_{1}-\frac{1}{N}, x_{2}+\frac{1}{N}, x_{3}, x_{4}\right)$. Such a mutation would occur at a rate $\mu x_{1}$. Therefore the propensity function (defined here as the expected number of transitions per unit time) for this transition would equal the transition rate times the population size:

$$
a\left(x_{1}-\frac{1}{N}, x_{2}+\frac{1}{N}, x_{3}, x_{4} \mid x_{1}, x_{2}, x_{3}, x_{4}\right)=\mu x_{1} N
$$

In general, the propensity functions due to mutations for all transitions can be written as:

$$
\begin{aligned}
& a\left(x_{i}-\frac{1}{N}, x_{j}+\frac{1}{N}, x_{k}, x_{l} \mid x_{i}, x_{j}, x_{k}, x_{l}\right) \\
& \quad= \begin{cases}0, & \text { if }\{i, j\} \text { is }\{1,4\} \text { or }\{2,3\} \\
\mu x_{i} N, & \text { otherwise }\end{cases}
\end{aligned}
$$

\section{SELECTION}

We assume that tag-carriers interact with other tag carriers in a prisoner's dilemma game, whereas tagless individuals never interact. This model setup is slightly different from previous studies, which assumed that all individuals carry one or the other tag, and interact with others possessing a similar tag. However, our setup may account for tags such as adhesion, because adhesive (tagcarrying) individuals are expected to form groups, whereas nonadhesive (tagless) individuals typically remain solitary. In case of tags that involve active signaling, we justify our setup as follows. The basic premise of tag-based cooperation is that all individuals inherently associate the tag with cooperation, that is a tag-carrying partner would be presumed to be a potential cooperator. Therefore, both cooperators and defectors, with or without the tag, prefer to interact only with individuals with tag, leaving tagless individuals noninteracting. We derive pay-offs for each of the four strategies under these assumptions.

In a pairwise prisoner's dilemma game, cooperators confer a benefit $b$ onto their partner and suffer a cost $c$ for their act. Defectors receive a benefit $b$ if their partner is a cooperator, but neither confer any benefit to cooperators nor suffer any cost. Tagless cooperators are also assumed to suffer a $\operatorname{cost} c$, whereas tagless defectors get zero payoff. Thus, our model assumptions make cooperators worse off - they do not get benefit from their own acts and continue to pay a cost even when solitary and not actively cooperating. Later, we consider scenarios with less stringent conditions, via public goods game and by assuming lack of cost for solitary cooperators.

Next, we assume that the subpopulation of tag-carriers is well-mixed, and generalize the pairwise prisoner's dilemma payoffs to the whole sub-population. It can be shown that in a group of size $n$ with $k$ cooperators, the average generalized payoff to cooperators is $(k-1) b /(n-1)-c$, and that to defectors is $k b /(n-1)$. Further, we assume that tag-carrying individuals suffer a $\cos t c_{T}$ associated with the tag. Thus, we can write the average payoffs received by the different types of individuals (notations as per Fig. 1) as follows

$$
V_{1}=V_{S C}=1-\alpha c
$$

$$
\begin{gathered}
V_{2}=V_{T C}=1+\alpha\left(\left(\frac{p_{T} N_{T}-1}{N_{T}-1}\right) b-c-c_{T}\right) \\
\approx 1+\alpha\left(p_{T} b-\frac{b}{N_{T}}-c-c_{T}\right) \\
V_{3}=V_{S D}=1
\end{gathered}
$$

$$
\begin{aligned}
V_{4}=V_{T D} & =1+\alpha\left(\frac{p_{T} N_{T}}{N_{T}-1} b-c_{T}\right) \\
& \approx 1+\alpha\left(p_{T} b-c_{T}\right)
\end{aligned}
$$

where $\alpha$ determines the strength of selection, $N_{T}$ is the number of tag-carriers $\left(N_{T}=\left(x_{2}+x_{4}\right) N\right.$, assumed to be $\left.\gg 1\right)$, and $p_{T}$ is the proportion of cooperators within the tag-carrying subpopulation 
$\left(p_{T}=x_{2} /\left(x_{2}+x_{4}\right)\right)$. The average payoffs of tag-carrying and tagless individuals are

$$
\begin{gathered}
V_{T}=p_{T} V_{T C}+\left(1-p_{T}\right) V_{T D}=1+\alpha\left[p_{T}\left(b-\frac{b}{N_{T}}-c\right)-c_{T}\right] \\
V_{S}=p_{S} V_{S C}+\left(1-p_{S}\right) V_{S D}=1+\alpha\left(-c p_{S}\right)
\end{gathered}
$$

where $p_{S}$ is the proportion of tagless individuals $\left(p_{S}=x_{1} /\right.$ $\left.\left(x_{1}+x_{3}\right)\right)$.

We assume that individuals die at a rate $d$. When an individual dies, it is replaced by the offspring of one of the remaining individuals. The parent individual is chosen with a probability proportional to its payoff. Since we assumed a well-mixed population with $N_{T} \gg 1$, the above payoffs are almost identical under either death-birth updating or birth-death updating (Zukewich et al. 2013). When the dead individual is replaced by another with a different type, the system state will change. For example, the probability for the transition $\left(x_{1}, x_{2}, x_{3}, x_{4}\right) \rightarrow$ $\left(x_{1}-\frac{1}{N}, x_{2}+\frac{1}{N}, x_{3}, x_{4}\right)$ as in equation (1) will be the probability that a tagless cooperator dies $\left(=d x_{1}\right)$ times the probability that it is replaced by a tag-carrying cooperator (assuming a Moran process, this probability equals $x_{2} V_{2} / \bar{V}$, where $\bar{V}$ is the average payoff of all individuals). Then, the total propensity for this transition due to both selection and mutation can be written as

$$
\begin{aligned}
& a\left(x_{1}-\frac{1}{N}, x_{2}+\frac{1}{N}, x_{3}, x_{4} \mid x_{1}, x_{2}, x_{3}, x_{4}\right) \\
& \quad=\mu x_{1} N+d x_{1} x_{2} \frac{V_{2}}{\bar{V}} N
\end{aligned}
$$

where $\bar{V}=\sum x_{i} V_{i}$. Similarly, we can write all other propensity functions as follows.

$$
\begin{aligned}
& a\left(x_{i}-\frac{1}{N}, x_{j}+\frac{1}{N}, x_{k}, x_{l} \mid x_{i}, x_{j}, x_{k}, x_{l}\right) \\
& = \begin{cases}N d x_{i} x_{j} V_{j} / \bar{V}, & \text { if }\{i, j\} \text { is }\{1,4\} \text { or }\{2,3\} \\
N d x_{i} x_{j} V_{j} / \bar{V}+\mu x_{i} N, & \text { otherwise }\end{cases}
\end{aligned}
$$

Further, if we assume that selection is weak, then $\alpha \rightarrow 0$ and $\bar{V} \approx 1$.

\section{DRIFT}

Since the population size is finite, the system is expected to experience demographic stochasticity, or drift. We write the chemical Langevin equation (Gillespie, 2000) for the system as follows:

$$
d \boldsymbol{X}(t)=\boldsymbol{\Delta} \boldsymbol{A} d t+\frac{1}{\sqrt{N}} \boldsymbol{\Delta} \sqrt{\operatorname{diag}(\boldsymbol{A})} \boldsymbol{\eta}(t) \sqrt{d t}
$$

where, if there are $N$ species and $M$ transition channels, $\boldsymbol{X}$ is a random variable whose instantaneous value is the system state $\boldsymbol{x}, \boldsymbol{A}_{M \times 1}$ is the vector of transition rates in each channel, $\boldsymbol{\Delta}_{N \times M}$ contains the change in the number of individuals of each species due to a transition in each channel, and $\eta_{M \times 1}$ is an $M$ dimensional Gaussian random variable. This equation is interpreted in an Itosense. See Supplementary Information for derivation. The noise term in equation (11) contains a factor of $1 / \sqrt{N}$, which accounts for demographic stochasticity: larger the population size, smaller the noise term, therefore, smaller the drift.

\section{Transformation of variables}

To make our model more intuitive, we derive the dynamics of the system by using a different but equivalent set of state variables: the proportion of cooperators within the tag-carrying and tagless subpopulations ( $p_{T}$ and $p_{S}$, respectively) and the total proportion of tag-carriers in the population $(q)$. This set $\left\{p_{T}, p_{S}, q\right\}$ is more intuitive because it allows us to visualize the dynamics of selection within and across the two (tag-carrying and tagless) subpopulations.

\section{Results}

\section{A COMBINED SELECTION-MUTATION-DRIFT EQUATION}

After a change of variables in equation (11), we get the final mutation-selection-drift equation that describes the dynamics of subpopulations of tag-carrying cooperators $\left(p_{T}\right)$, solitary cooperators $\left(p_{S}\right)$, and total proportion of tag-carriers $(q)$ as

$$
\begin{aligned}
& {\left[\begin{array}{c}
d p_{T} \\
d p_{S} \\
d q
\end{array}\right]=\alpha d\left[\begin{array}{c}
-\left(c+\frac{b}{q N}\right) p_{T}\left(1-p_{T}\right) \\
-c p_{S}\left(1-p_{S}\right) \\
q(1-q)\left(p_{T}\left(b-\frac{b}{q N}-c\right)+c p_{S}-c_{T}\right)
\end{array}\right] d t} \\
& +\mu\left[\begin{array}{c}
1-2 p_{T}+\frac{1-q}{q}\left(p_{S}-p_{T}\right)\left(1-\frac{1}{q N}\right) \\
1-2 p_{S}+\frac{q}{1-q}\left(p_{T}-p_{S}\right)\left(1-\frac{1}{(1-q) N}\right) \\
1-2 q
\end{array}\right] d t \\
& +\sqrt{\frac{1}{N}}\left[\begin{array}{cccc}
0 & \frac{1-p_{T}}{q} & 0 & -\frac{p_{T}}{q} \\
\frac{1-p_{S}}{1-q} & 0 & -\frac{p_{S}}{1-q} & 0 \\
0 & 1 & 0 & 1
\end{array}\right] \boldsymbol{\Delta} \sqrt{\operatorname{diag}(\boldsymbol{A})} \boldsymbol{\eta}(t) \sqrt{d t}
\end{aligned}
$$

The first matrix-term on the right-hand side describes selection, the second matrix term describes the deterministic macroscopic effects of mutations, and the third term describes the effects of demographic noise.

This system of equations reveals two key features of the mutation-selection dynamics. Firstly, the first matrix-term reveals that cooperators in both tag-carrying $\left(p_{T}\right)$ and tag-less $\left(p_{S}\right)$ 
subpopulations are always under negative selection (Fig. 3 $\mathrm{B}(\mathrm{b}, \mathrm{c}, \mathrm{d}))$. On the other hand, selection for tag-carriers depends on the proportion of tag-carrying cooperators and tagless cooperators: when $p_{T}$ is high (when $p_{T}>\left(c_{T}-c p_{S}\right) /\left(b-\frac{b}{q N}-c\right)$ ), there is strong selection for tag-carriers (Fig. $3 \mathrm{~B}(\mathrm{c})$ ), whereas when $p_{T}$ falls below this threshold, there is strong selection for tagless individuals (Fig. 3 B(e)).

Secondly, $q$ appears in the denominator of the mutation term. This implies that when the proportion of the tag-carrying subpopulation is very low ( $q \approx 0$ ), mutations cause large changes in the proportion of cooperators within that subpopulation $\left(p_{T}\right)$ (Fig. 3 $\mathrm{B}(\mathrm{a}, \mathrm{b}))$. Similar changes happen in the proportion of cooperators within the tagless subpopulation, when most of the population consists of tag-carriers $(q \approx 1)$.

The two key parameters of the coevolutionary model are the cost of cooperation $(c)$ and the cost of the $\operatorname{tag}\left(c_{T}\right)$. These values determine the threshold that tips the direction of selection in favor of the tag. Specifically, selection will favor tag carriers when $p_{T}>\left(c_{T}-c p_{S}\right) /(b-c)$. If $c / b \ll 1$, then the condition approximately reduces to $p_{T}>c_{T} / b$. The other two parameters $\mu$ and $N$ determine the strength of mutations and noise. Typically in nature, the mutation rate is small. We assume that $\mu$ is of the order of $10^{-6}$. Noise is large in small populations (small $N$ ), and goes to zero as $N \rightarrow \infty$. In this case, equation (12) reduces to the replicator dynamics equation, which is an ordinary differential equation.

Put together, the coevolutionary dynamics described by equation (12) suggests that mutation and selection alone can maintain very low levels of cooperation, but demographic noise can facilitate a net increase in tag-based cooperation when certain threshold conditions are satisfied. We explore this in detail via further analyses and numerical simulations of equation (12) below.

\section{INFINITE POPULATIONS CANNOT SUPPORT TAG-BASED COOPERATION}

First, we explore the coevolutionary dynamics in equation (12) without mutations. The fixed points of the system are $p_{T}^{*}=\{0,1\}$, $p_{S}^{*}=\{0,1\}$, and $q^{*}=\{0,1\}$, that is any of the six combinations of these values of $p_{T}, p_{S}$, and $q$ is a fixed point (such as $\left(p_{T}, p_{S}, q\right)=(0,0,0),(0,1,0),(0,0,1)$ etc $)$. Of these, the only stable fixed point is $p_{T}^{*}=p_{S}^{*}=q^{*}=0$. Thus, the deterministic equilibrium of the system without mutations does not allow either cooperation or the phenotypic tag to persist. This is expected, as both the traits are costly.

Next, we consider the effect of mutations in infinite populations, that is, in the absence of drift and demographic noise. In this case, the proportion of cooperators and tag-carriers will be a result of mutation-selection equilibrium. In general, the nonneg-

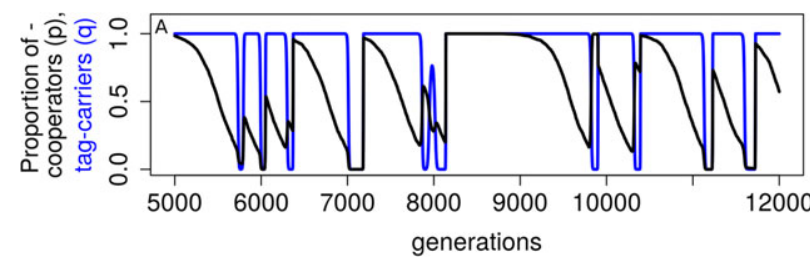

Figure 2. Demographic noise together with a cost of tag induces coevolutionary cycling. (A) The proportion of cooperators and proportion of tag-carriers both show cyclical dynamics, and the population switches rapidly between tag-carrying and tagless states. Loss of the tag (i.e., a population switch to tagless state) is preceded by a decline in cooperator frequency. Similarly, recovery of the tag is preceded by an emergence of a few tag-carrying cooperator mutants. See Figure 3 for the mechanism behind this "cyclic" dynamic.

ative real fixed points (other complex roots exist, which are not listed here) of equation (12) are

$$
\begin{aligned}
p_{T}^{*} & =\left\{\frac{c_{T}-c}{b}, \frac{c_{T}+c}{b}\right\} \\
p_{S}^{*} & =\left\{\frac{c\left(c_{T}-c\right)-\mu\left(b+c-c_{T}\right)}{c\left(c_{T}-c\right)-b \mu}, \mu \frac{\left(b-c-c_{T}\right)}{c\left(b-c-c_{T}\right)+b \mu}\right\} \\
& \approx\left\{1-\mu \frac{\left(b+c-c_{T}\right)}{c\left(c_{T}-c\right)}, \frac{\mu}{c}\right\} \\
q^{*} & =\mu \frac{b}{c\left(c_{T}-c\right)}
\end{aligned}
$$

For very small mutation rates $\left(\mu \sim 10^{-6}\right), p_{S}^{*} \approx\{1,0\}$, and $q^{*} \approx 0$. Thus, $p^{*}=p_{T}^{*} q^{*}+p_{S}^{*}\left(1-q^{*}\right) \approx 0$. To summarize, in the absence of demographic noise, but with realistic mutation rates, the fixed points are nonzero, but extremely small. This results in negligible evolved proportions of both cooperators and tag-carriers. Thus, when population size is infinite, neither mutations nor selection can support tag-based cooperation.

\section{DEMOGRAPHIC NOISE INDUCES PHENOTYPE SWITCHING AND PROMOTES TAG-BASED COOPERATION}

Now, let us consider the case of finite population size, where demographic noise becomes important. Equation (12) is now a system of coupled nonlinear stochastic differential equations, and can no longer be solved analytically. We simulate the system numerically using Ito interpretation. We find cyclical dynamics of both traits, that is the proportion of cooperators $(p)$ and the proportion of tag-carriers $(q)$. We observe (Fig. 2 A; Fig. S1) that the timescale for changes in $q$ is much greater than that of changes in $p$ - the population switches rapidly between tag-carrying and tagless states. This is due to the strong difference in payoffs of tagcarrying and tagless individuals. The proportions of cooperators among both tag-carrying and tagless sub-populations ( $p_{T}$ and $p_{S}$ ) fluctuate when the respective populations are low. 


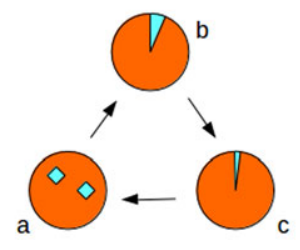

A No tag-based interactions

Figure 3. Schematic of how cycles arise due to demographic noise and coevolutionary dynamics in a population where interactions are conditional (tag-based). (A) Cooperators cannot sustain when there are no tags, that is interactions are unconditional: (a) Cooperative mutants (cyan diamonds) arise in a well-mixed population of defectors (red). (b) These mutants cause a small change in the frequency of cooperators (cyan sector). (c) Selection eliminates them as cooperation is costly. Thus, with unconditional interactions or in well-mixed populations, the frequency of cooperators does not exceed the level maintained by mutations alone. (B) Cooperators and tag-carriers both continuously appear and disappear when interactions are conditional on the tag: (a) Cooperative mutants arise in the small subpopulation of tag-carrying individuals (left). (b) This increases the frequency of cooperators within the tagcarrying subpopulation. (c) This small subpopulation grows faster relative to the non-tag-carrying subpopulation (right), causing the overall proportion of cooperators to increase. (d) Selection at the level of individuals simultaneously reduces the proportion of cooperators in both subpopulations. (e) Finally, with no cooperative benefits, the tag-carrying subpopulation drops relative the tagless subpopulation. The cycle then repeats. On average, the frequency of cooperators can be much higher than what can be maintained by mutations alone, or in the case of a costless tag.

We illustrate with an example. Suppose that in a total population of $N=10^{6}$ individuals, there are five tag-carrying individuals $\left(q=5 \times 10^{-6}\right)$, all of whom are defectors. Thus, $p_{T}=0$. If a mutant tag-carrying cooperator now appears in this population, such as by mutation from a tagless cooperator, $p_{T}$ becomes 0.2 , which is a large change in a single timestep. Compare this with similar changes in the tagless population. Such a mutation would change $p_{S}$ by $\sim 10^{-6}$. In equation (12), this effect is captured by the presence of $q$ and $1-q$ in the denominators of the noise terms. Figure $3 \mathrm{~B}$ schematically shows how cycles arise in the system. First, when the population is tagless, a few tag-carrying cooperator mutants quickly lead the entire population to be tagcarrying. Similarly, when the population is tag-carrying, a decline in cooperator frequency quickly leads to loss of the tag in the entire population.

To ensure that our results from simulations of equation (11) are not artefacts of the assumptions of the chemical Langevin equations, we compare the timeseries in Figure 2 with that obtained by simulating our microscopic model using the Gillespie algorithm (Fig. S3B). We find qualitative agreement in the results using both the methods. However, since the two methods were simulated at different population sizes $(N \sim 100$ for Gillespie simulations and $N \sim 10^{6}$ for the chemical Langevin equation), quantitative comparison of these results is difficult.

\section{COOPERATORS THRIVE AT INTERMEDIATE COSTS OF TAG}

When the cost of the tag is zero, all individuals evolve the tag. Thus, even though the proportion of tag carriers becomes one, defectors are easily able to invade cooperators, causing the proportion of cooperators to go to zero. When the cost of the tag is very high, the benefits of cooperation are not able to offset the cost of the tag. Therefore, again, cooperation does not evolve. For intermediate cost of the tag, cooperation can sustain (Fig. 4B). As intuitively expected, the proportion of cooperators increases with decreasing cost of cooperation (Fig. 4A). As the cost of cooperation decreases, the switching frequency decreases. For very low costs of cooperation, the population is almost at equilibrium (very low frequency of switching) with a high proportion (>95\%) of tag-carrying cooperators (Fig. 4C). The frequency of switching peaks at intermediate costs of the tag (Fig. 4C,F). Furthermore, we find that greater noise (arising from lower population size) allows cooperation to sustain for higher costs of cooperation (Fig. 4DF). Both proportions increase with increasing noise, and decrease with mutation rate (Fig. 5).

\section{COMPARISON WITH NULL MODELS}

We showed that in our system, tag-based interactions, cost of tag, demographic noise, and mutations are the key features that enable the evolution of cooperation. Absence of one or more of these features is a null scenario. We compare the evolved values of cooperation in these null scenarios. First, in the absence of tag-based interactions, any individual can interact with any other individual with equal probability. In such a population, either all individuals are solitary or all individuals form a single large group. In either case, the population is well-mixed. We simulated a well-mixed population of cooperators and defectors where the next generation is sampled with a probability proportional to individual fitness and with mutations, and found the evolved proportion of cooperators to be relatively small (for parameters as in Fig. S1, the evolved proportion of cooperators without tags is just 0.2 , against 0.66 in the presence of tags). Second, if the tag incurs no cost $\left(c_{T}=0\right)$, all individuals evolve the tag $(q \approx 1)$ and the proportion of cooperators remains low (see left edge in the panels of Fig. 4). Third, in the infinite population limit (demographic noise $\rightarrow 0$ ), the evolved levels of cooperation due to mutation-selection equilibrium (eq. 13) are much lower (of the 

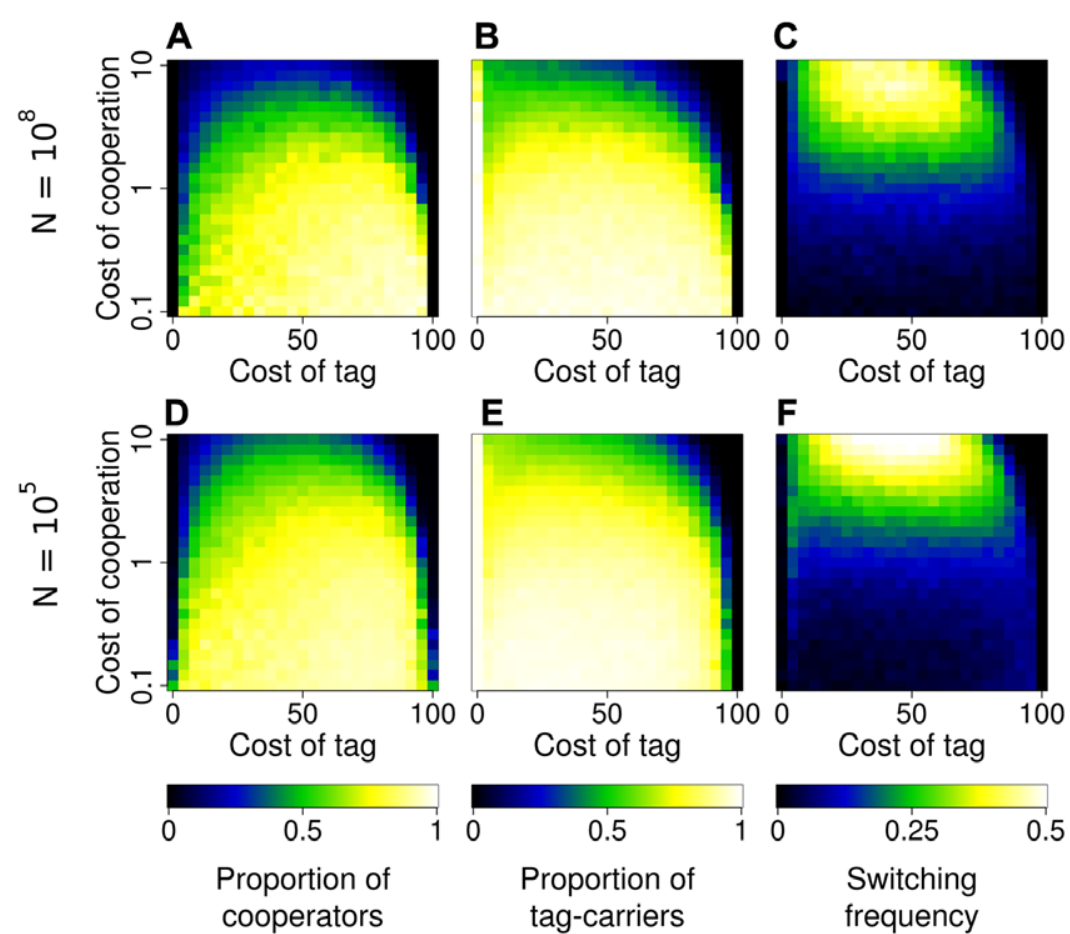

E

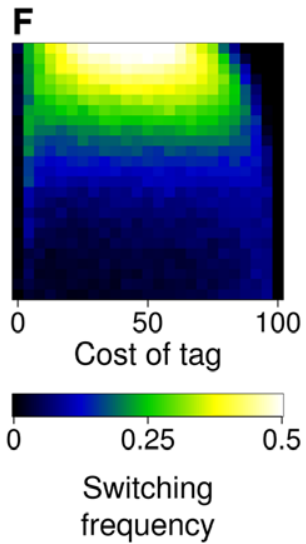

Figure 4. Cooperators thrive at low costs of cooperation, intermediate costs of tag, and lower population sizes (A) The average proportion of cooperators and tag-carriers both decrease with the cost of cooperation $(A, B)$. The proportion of cooperators is zero when the cost of the tag is either zero or too high, and is maximum for an intermediate cost of cohesion (A). The proportion of tag-carriers decreases with the cost of the tag (B). The average proportion of cooperators and tag-carriers is close to one for a very low cost of cooperation. However, as the cost of cooperation increases, the frequency with which the population switches between tag-carrying and tagless states increases, leading to lowering of both the average traits (C). For a given cost of cooperation, increased noise (due to lower population size) leads to a greater proportion of cooperators and tag-carriers (D-F, also see Fig. 5D-F). Parameter values: $\mu=2 \times 10^{-6}$, $N=10^{5}, 10^{8}$. Other parameters as in Table S2.

order of the mutation rate). Finally, in the absence of mutations, a population of solitary and tag-less defectors remains stable, with no possibility for the emergence of tag-carrying cooperators-a necessary condition for the emergence of cycles observed in our simulations. Even if we did start with a few tag-carrying cooperators, it will go through one cycle of increased proportions of cooperators and tag-carriers but eventually face a decline of the proportion of (tag-carrying) cooperators.

\section{MODEL VARIATIONS}

\section{Coevolutionary dynamics in the public goods game}

So far, we used the prisoner's dilemma game to model cooperative interactions. In this section, we consider a model variation: we explore the coevolutionary dynamics using the public goods game, which models weaker forms of cooperation but is relevant for a number of biological contexts. In the public goods game, cooperators produce a benefit $b$ that is shared by all individuals, including the producer. Defectors partake of the benefit, but don't contribute in return. Thus, in the tag-carrying subpopulation, the payoff to cooperators is $V_{t c}=1+\alpha\left(p_{T} b-c-c_{T}\right)$, and that to defectors is $V_{t d}=\alpha\left(p_{T} b-c_{T}\right)$. Plugging these values in equation (12), the selection terms in equation (12) become

$$
\left[\begin{array}{c}
d p_{T} \\
d p_{S} \\
d q
\end{array}\right]_{\mathrm{sel}}=\alpha d\left[\begin{array}{c}
-c p_{T}\left(1-p_{T}\right) \\
-c p_{S}\left(1-p_{S}\right) \\
q(1-q)\left(p_{T}(b-c)+c p_{S}-c_{T}\right)
\end{array}\right] d t
$$

In the above equation, the features that lead to cyclical dynamics as shown earlier remain unchanged: selection for cooperators is negative in both subpopulations, but selection for tagcarrying individuals depends on the threshold value of $p_{T}$ and $p_{S}$. Indeed, our results hold true in the PGG game (Fig. S2).

\section{Evolutionary dynamics with two tags}

For all our main results, we considered a single tag that facilitates interactions, whereas tagless individuals remain solitary. This model is especially suited to model cellular adhesion and signalling. However, this model differs slightly from most previous studies on tag-based cooperation, which consider that each individual carries a certain tag, and individuals interact with 


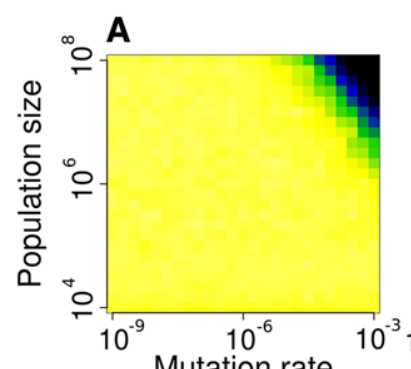

B
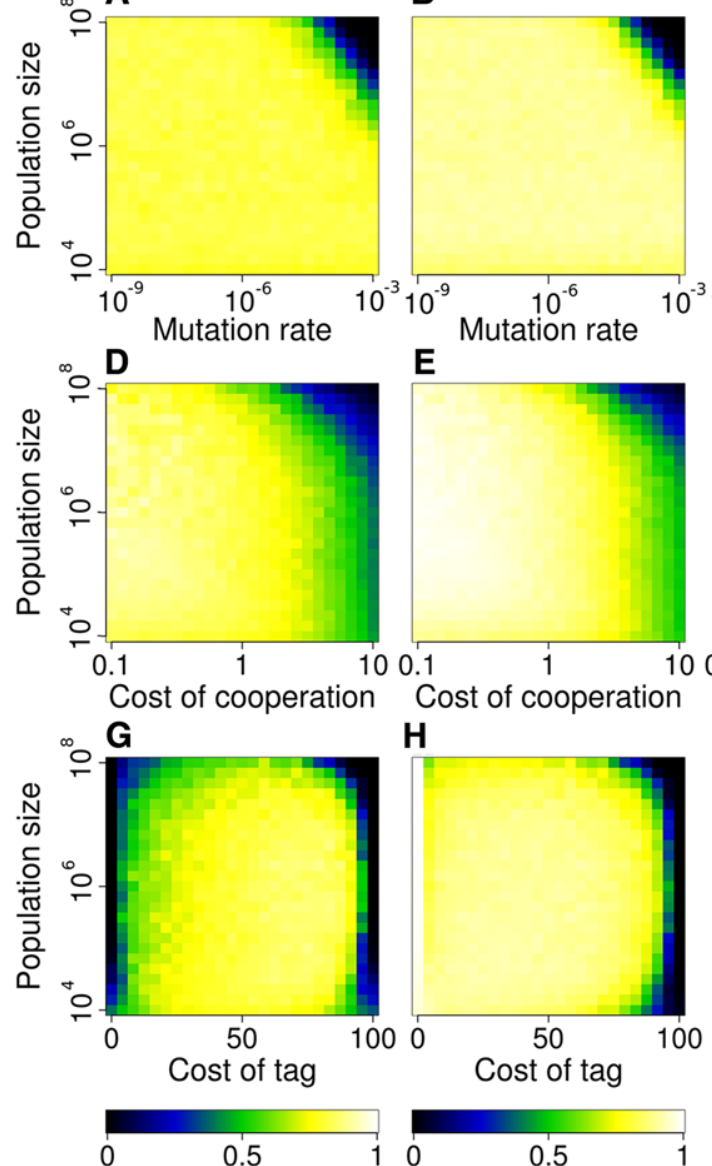

Proportion of cooperators
E

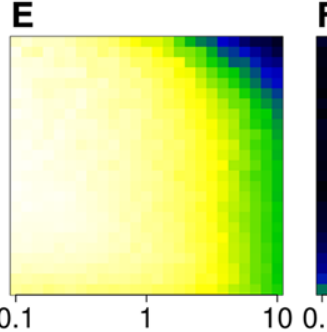

Cost of cooperation H

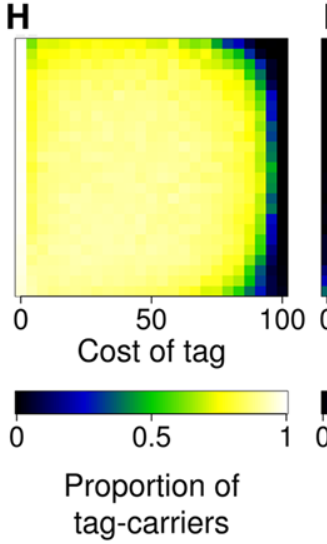

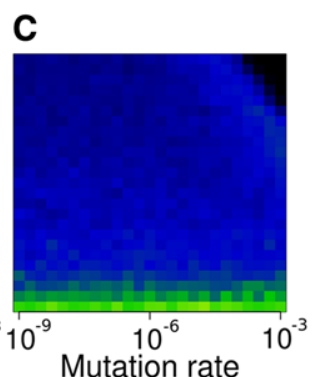

$\mathbf{F}$

Mutation rate

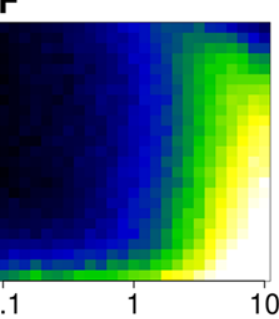

Cost of cooperation

I

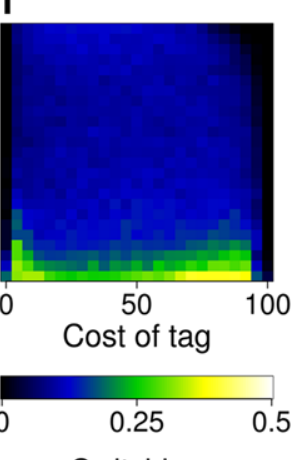

Switching

frequency

Figure 5. Cooperators sustain over several orders of magnitude of cost of cooperation, mutation rate, and population size. For a given parameter set, the average proportions of cooperators and tag-carriers both increase with decreasing population size (A-I), but decrease with mutation (A-C) and cost of cohesion (D-F); and first increase, reach a maximum, and sharply drop to zero with increasing cost of $\operatorname{tag}(\mathrm{G}-\mathrm{I})$. Parameter values: $\mu=2 \times 10^{-5}, c=1, c_{T}=50$, unless varied on any axis. Other parameters as in Table S2.

others with a similar tag. To facilitate comparison with those studies, here we extend our model to consider two tags A and B, such that interactions now happen between $\mathrm{A}-\mathrm{A}$ individuals or $\mathrm{B}-\mathrm{B}$ individuals, but not A-B individuals. Now, $q$ refers to the proportion of A-individuals, and $c_{T}$ refers to the cost of having tag A relative to that having tag $\mathrm{B}$. With these assumptions, the selection terms in equation (12) become

$$
\left.\begin{array}{c}
{\left[\begin{array}{c}
d p_{A} \\
d p_{B} \\
d q
\end{array}\right]_{\mathrm{sel}}} \\
-\left(c+b / N_{A}\right) p_{A}\left(1-p_{A}\right) \\
-\left(c+b / N_{B}\right) p_{B}\left(1-p_{B}\right) \\
q(1-q)\left(\left(p_{A}-p_{B}\right)(b-c)-c_{T}\right)-\frac{b}{N}\left((1-q) p_{A}-q p_{B}\right)
\end{array}\right] d t
$$

The mutation and drift terms remain identical to equation (12) as they do not involve individual payoff terms.

We simulate this system for $c_{T}=0$ and $c_{T}= \pm 25$, and find similar cyclical dynamics (Fig. S4). The case $c_{T}=0$ essentially reproduces the dynamics from previous studies on chromodynamics, and we in fact find a high proportion of cooperators in this case. We speculate that in a system with more than two tags also, cooperation would be favoured via cycles in the each individual tag's proportion.

\section{Dynamics when strategy and tag mutation rates are different}

For analytical tractability, we had so far assumed that the rate at which individuals switch strategy (cooperative to defective and vice versa) is the same as the rate at which they switch between tag-carrying and tagless states. In reality, the two mutation rates 
may be different. We find similar cyclical dynamics of the tag in a model where two different mutation rates are considered (Fig. S5).

\section{Dynamics when solitary cooperators suffer no cost}

Thus far, we assumed that solitary (tagless) cooperators also suffer a cost $c$. This models a strong form of cooperation, but in realistic settings, cooperators may suffer a cost only when they interact. We simulate the prisoner's dilemma scenario where solitary cooperators get zero payoff, and find that our results are robust to this variation (Fig. S6).

\section{Discussion}

We showed that demographic noise coupled with coevolutionary dynamics of costly cooperation and a costly tag can facilitate the evolution of tag-based (greenbeard) cooperation. We found that the cost of the tag can lead to cyclical dynamics where the tag is continuously lost and rediscovered, which is reminiscent of many phenotype-switching organisms. Therefore, the cost of tag together with demographic noise, both of which are relevant for natural populations, may offer a possible solution to the problem of instability of tag-based cooperation. We demonstrated the robustness of our results over a wide range of population sizes, mutation rates, cost-benefit parameters, and for both strong and weak forms of cooperation. This renders our model potentially applicable to a diverse range of ecological systems, including adhesive or quorum sensing microbes and cohesive groups of organisms. Our proposed mechanism is similar to some of the previously proposed mechanisms based on beard-chromodynamics, but differs in one crucial aspect: whereas beard-chromodynamics (in the absence of cost of tag) relies heavily on the availability of multiple tags, our mechanism allows for continuous rediscovery of a single tag, as long as it is costly.

\section{EMPIRICAL APPLICATIONS}

\section{Model assumptions, mechanism, and applicability}

In our simulations, the population starts with all tagless defectors. The emergence of a few tag-carrying cooperators, via mutations in both traits, is crucial to kickstart selection for cooperators. However, we emphasize that mutations in both traits need not be simultaneous. Rather, it is sufficient that mutants survive until a mutation in the second trait appears. In a large population under weak selection, as shown in our results (e.g., Fig. 2), this is feasible. In small populations (such as in Fig. S3), however, a high mutation rate is necessary to achieve the necessary mutations. Both scenarios (low $\mu$ with high $N$, and high $\mu$ with low $N$ ) are biologically feasible: the former may be suited for bacterial populations, whereas the latter, for example, for cultural evolution.
We assumed a weak selection and a constant population size in our simulations. We speculate that relaxing these assumptions is likely to favor cooperators in some cases. For instance, if the selection is strong, then the population size will change (increase or decrease) depending on the average population fitness. If we begin with a low population size, the strength of drift may overcome negative selection on cooperators. Increase in cooperator frequency in the population results in higher benefits of cooperation. Hence the fitness of each individual, and consequently the average population fitness, will be higher. This in turn may lead to larger population sizes until the effects of competition stabilize the population size. Indeed, previous studies have argued that allowing population sizes to change, a biologically realistic feature, may facilitate the evolution of cooperators (Brockhurst 2007; Huang et al. 2015; Constable et al. 2016).

Many examples of cooperation in nature can be described either by a prisoner's dilemma game, or a public goods game. Prisoner's dilemma is a strong form of cooperation/altruism, where cooperators do not benefit from their own cooperative act. On the other hand, public goods cooperation is often referred to as weak altruism, because cooperators share a part of the benefit they produce (West et al. 2007). Our model formulation applies equally well to both types of cooperation (Fig. 4 and Fig. S2). This extends its applicability to organisms across taxa, especially in the microbial world. We discuss specific empirical examples below.

\section{Evolution of adhesion, life cycles, and multicellularity}

Many microbes secrete adhesive molecules that allow them to stick to other cells or to substrates (Cooksey and Wigglesworth 1995; Lodish et al. 2000; Dunne 2002). Adhesion can be considered as a tag in our model, because in a well-mixed medium, adhesive cells automatically segregate from non-adhesive cells (Drescher et al. 2014; Joshi et al. 2017). Cooperation is also widespread among these microbes, such as in the production of biofilms for antibiotic resistance (Kreft and Bonhoeffer 2005; Xavier and Foster 2007; Nadell et al. 2009), self-destruction by cells to provide nutrients to neighboring cells (Ackermann et al. 2008), or cooperative migration of cancer cells during metastasis (Deisboeck and Couzin 2009). In fact, cancer cells are also known to switch between individual and collective migration during metastasis (Sahai 2005). Our proposed mechanism may explain not only how adhesion and cooperation may emerge simultaneously in a variety of microbes, but also how noise and coevolutionary dynamics may lead to the phenotype switching behavior observed in organisms such as cancer cells.

It is argued that the first step in the evolution of multicellularity is the origin of simple multicellular clusters from single cells (Smith and Szathmary 1997). The formation of stable clusters (in which cells stick to each other by secreting adhesive 
molecules) requires that individual cells cooperate to maximize the fitness of the cluster over solitary cells. This would then set the stage for multicellular clusters to become units of selection, where their fitness becomes decoupled from the fitness of constituent cells (Libby and Rainey 2013; Hammerschmidt et al. 2014). Such multicellular clusters readily evolve in laboratory conditions (Koschwanez et al. 2011; Ratcliff et al. 2012). However, they are often short-lived because they are susceptible to invasion by mutant cheaters. Our model suggests that proliferation of cheaters may be prevented by mutations and demographic noise (with adhesion as the greenbeard), potentially allowing clustering, and cooperation to emerge simultaneously. Furthermore, it is thought that an intermediate step in the evolution of multicellularity is a life cycle with unicellular and multicellular stages (Libby and Rainey 2013). The switching behavior observed in our model can also be thought of as a primitive life cycle, where the tagless phase corresponds to a unicellular stage and the tagcarrying phase corresponds to the multicellular stage.

\section{Evolution of quorum sensing}

Quorum sensing cells communicate with other cells via signaling molecules (Waters and Bassler 2005; Keller and Surette 2006; Nadell et al. 2009). The uptake of the signaling molecules by cells induces them to produce more signaling molecules, a process called auto-induction (Diggle et al. 2007). If sufficient number of signaling cells are present, the signal level increases via such positive feedback. When the signal crosses a threshold level, cells secrete public goods, such as constituents of a biofilm. A vast amount of literature on quorum sensing has focused on the proximate mechanisms and responses of cell-cell signaling (Miller and Bassler 2001). Yet, the evolutionary mechanisms that enable the (possibly synergistic) emergence of signaling and cooperation remain poorly understood, and only a few studies have attempted to study the evolution of quorum sensing using a game theoretic framework (e.g., Czárán and Hoekstra 2009). These studies conclude that spatial structure and limited dispersal are crucial for the evolution of quorum sensing controlled cooperation.

Based on our results, we speculate that demographic noise may allow quorum sensing to evolve even in the absence of local dispersal. Our model provides a minimal analytical framework that can be adapted to study the evolution of quorum sensing. The ability to produce and recognize signaling molecules can be considered as a tag in our model. Cells that produce both the signal and the public good, correspond to tag-carrying cooperators in our model. They are susceptible to exploitation by several types of cheaters, such as those that produce the signal but do not cooperate (corresponding to tag-carrying defectors), or those who cooperate but do not produce the signal (corresponding to tagless cooperators) (Diggle et al. 2007), or those who neither produce the signal nor the public good (corresponding to tagless

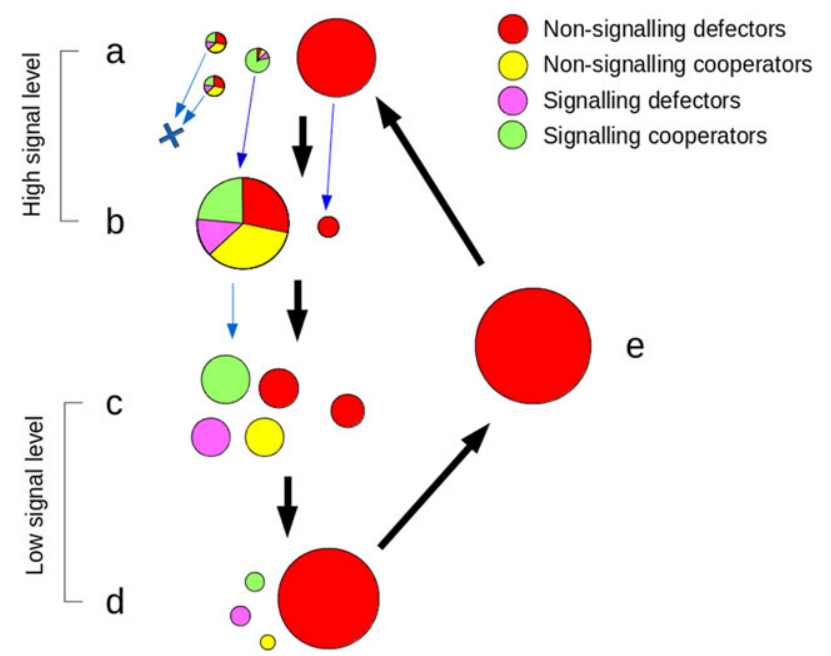

Figure 6. Schematic of how our model framework may be applied to quorum sensing. Mutations lead to a large fraction of signaling cooperators in one of the small subpopulations (A). While other subpopulations die off, this subpopulation rapidly increases in size (B). However, within the subpopulation, defectors begin to increase, leading to a decrease in signaling cooperators. Therefore, the signal level drops, and individuals disperse (C). Nonsignaling defectors invade the population (D) and the cycle repeats again (E).

defectors). In such a system, clusters of signaling cooperators that have formed by random chance would remain stable and grow in size. Within these clusters, the three types of cheaters will eventually outcompete cooperators. Nonsignaling individuals may initially join such clusters and gain benefits, but since they do not produce the signal, the signal level in such clusters will eventually go down and individuals will disperse (Fig. 6). By incorporating these effects into our model, the dynamics of signal production and cooperation can be studied.

\section{COMPARISON WITH PREVIOUS STUDIES}

A number of studies have investigated the role of demographic noise in evolutionary contexts such as reciprocal cooperation (Imhof et al. 2005; Bladon et al. 2010), language dynamics (Fudenberg et al. 2006), exploration and social learning (Traulsen et al. 2009), and evolution of cooperation in fluctuating environments (Assaf et al. 2013). Here, we showed how demographic noise promotes cooperation in a system of tag-based interactions. Furthermore, we applied analytical techniques based on chemical Langevin equations to construct stochastic differential equations (SDE's) to analyze our model. We contrast this approach with the previous studies below.

Previous studies analyzing demographic noise in the evolution of cooperation have either used the Moran process-based analyses (Nowak et al. 2004; Imhof et al. 2005; Fudenberg et al. 2006; Traulsen and Nowak 2006, 2007; Traulsen et al. 2009), 
or used van Kampan's system size expansion method to derive Langevin equations (a system of SDEs) that describe the temporal evolution of state variables in a stochastic system (McKane and Newman 2005; Traulsen et al. 2006, 2009; Kogan et al. 2014). The former approach is useful to analytically derive the conditions for fixation of cooperation, but it requires a separation of mutation and selection timescales. The system-size expansion method does not require this assumption, but can be translated into the Langevin equations only if certain mathematical conditions are met. Our initial analyses, not presented in this article, showed that the system size expansion method was mathematically intractable to derive Langevin equations for our tag-based cooperation model. The chemical Langevin approach allows us to circumvent the mathematical difficulty by offering a simple heuristic to directly write down Langevin equations (Gillespie 2000). Although the chemical Langevin method has been widely used in chemistry, it has rarely been applied to equivalent problems in ecology and evolution. We think that there is immense scope for applying this method to investigate the role of stochasticity arising from finite sizes in many ecological and evolutionary contexts.

\section{EXTENSIONS}

We discuss some possible extensions of our model set up and analyses. We restricted ourselves to noniterative strategies. A substantial body of literature has established the importance of iterative strategies, such as tit-for-tat (Axelrod and Hamilton 1981) and win-stay-lose-shift (Nowak and Sigmund 1993), on the evolution of cooperation. Recognition is important for such strategies but most of that literature does not include the cost of recognition. It would be interesting to compare the levels of cooperation achieved in our model with those in repeated interactions.

As discussed above, cellular-adhesion and signaling mechanisms in cooperative systems can be viewed as tag-based cooperation. In these systems, group formation and collective movement of groups can play an important role in evolutionary outcomes. Several studies have indeed explored such effects by employing individual-based and spatially explicit modeling and simulation approaches (Powers et al. 2011; Garcia et al. 2014, 2015; Joshi et al. 2017). A promising direction for further research could be to examine the utility of, and to further extend, our modeling framework to include the effects of space and small group sizes.

A common metric computed in stochastic systems is the residence time in a particular state of interest. In Figs. 4-5, we have calculated the switching frequency $f_{S}$ of the proportion of tag-carriers. This can be related to the mean residence time in the tag-carrying state as $q / f_{S}$. The distribution of residence time in each state can in principle be calculated, and this would be an interesting direction to pursue in future studies.

Our model framework can also be readily modified to incorporate "spiteful" interactions, where the actor harms (i.e., im- poses a cost, say $\beta$, on) the recipient at a $\cos t c$ to self. Since both partners suffer a cost, it is considered to be difficult to evolve. Our equations may be reinterpreted to model the dynamics of spite, if we substitute $b=-\beta$ and $c_{T}=-\xi$. A negative $c_{T}$ implies that carrying the tag is beneficial for the individual, such as due to tag-linked benefits other than those due to cooperation. In such a case, our dynamical equation for tag-carriers becomes $d q=-q(1-q)\left(p_{T}\left(\beta-\beta / N_{T}+c\right)-c p_{S}-\xi\right)$, which is identical to equation (12), except for the difference in sign. However, further investigations are required to interpret these results, explore the role of various parameters including demographic noise in the evolution of spite behavior.

\section{Concluding Remarks}

In summary, our model provides insights into greenbeard cooperation based on analyses of a simple stochastic model using a novel analytical framework. We demonstrate that noise, arising from mutations and from a random sampling of interaction partners, induces qualitative changes in the evolutionary dynamics. We find cyclical behavior with an emergence and collapse of cooperation, where the deterministic equations alone would predict an equilibrium of defector population. We also demonstrated the importance of the cost of tag, a biologically realistic feature that is often ignored in previous studies, in facilitating cooperation. We present an analytical machinery based on chemical Langevin equations to analyze the effect of mutations and noise in the evolution of tag-based cooperation. Our model framework can be adapted to analyze diverse-related problems, including the evolution of grouping tendencies, cellular adhesion, life cycles, multicellularity, and quorum sensing.

\section{AUTHOR CONTRIBUTIONS}

J.J. designed the study and conducted the analyses, J.J. and V.G. synthesized the results and wrote the article, V.G. obtained funding.

\section{ACKNOWLEDGMENTS}

We would like to thank Jeffrey Philippson, Kavita Jain, and Appilineni Kushal for detailed and insightful comments on our methods and discussions on the chemical Langevin equation. VG acknowledges research support from DBT-IISc partnership program, DST Centre for Mathematical Biology at IISc Phase II (SR/S4/MS:799/12), infrastructure support from DST-FIST, and a partial support by the International Centre for Theoretical Sciences (ICTS) during a visit for participating in the program Third Bangalore School on Populational Genetics and Evolution (Code: ICTS/Prog-PGE2018/03).

\section{DATA ARCHIVAL}

All data used in this study are generated by simulations. The code for the simulations can be found at https://github.com/tee-lab/greenbeard_ cooperation. 


\section{LITERATURE CITED}

Ackermann, M., B. Stecher, N. E. Freed, P. Songhet, W. D. Hardt, and M. Doebeli. 2008. Self-destructive cooperation mediated by phenotypic noise. Nature 454:987-990.

Antal, T., H. Ohtsuki, J. Wakeley, P. D. Taylor, and M. A. Nowak. 2009. Evolution of cooperation by phenotypic similarity. Proc. Natl. Acad. Sci. 106:8597-8600.

Assaf, M., M. Mobilia, and E. Roberts. 2013. Cooperation dilemma in finite populations under fluctuating environments. Phys. Rev. Lett. 111:238101.

Axelrod, R., and W. D. Hamilton. 1981. The evolution of cooperation. Science 211:1390-1396.

Bladon, A. J., T. Galla, and A. J. McKane. 2010. Evolutionary dynamics, intrinsic noise, and cycles of cooperation. Phys. Rev. E 81:066122.

Brockhurst, M. A. 2007. Population bottlenecks promote cooperation in bacterial biofilms. PLoS ONE. 2:e634.

Caccamise, D. F., and D. W. Morrison. 1986. Avian communal roosting: implications of diurnal activity centers. Am. Nat. 128:191-198.

Constable, G. W. A., T. Rogers, A. J. McKane, and C. E. Tarnita. 2016. Demographic noise can reverse the direction of deterministic selection. Proc. Natl. Acad. Sci. 113:E4745-E4754.

Cooksey, L., and B. Wigglesworth. 1995. Adhesion of bacteria and diatoms to surfaces in the sea: a review. Aquatic Microbial Ecol. 09: 87-96.

Couzin, I. D., J. Krause, R. James, G. D. Ruxton, and N. R. Franks. 2002. Collective memory and spatial sorting in animal groups. J. Theoret. Biol. 218:1-11.

Czárán, T., and R. F. Hoekstra. 2009. Microbial communication, cooperation and cheating: quorum sensing drives the evolution of cooperation in bacteria. PLOS ONE 4:e6655.

Dawkins, R. 1976. The selfish gene. Oxford Univ. Press, Oxford.

Dawkins, R. 1982. The extended phenotype. Oxford Univ. Press, Oxford, England.

Deisboeck, T. S., and I. D. Couzin. 2009. Collective behavior in cancer cell populations. BioEssays 31:190-197.

Diggle, S. P., A. S. Griffin, G. S. Campbell, and S. A. West. 2007. Cooperation and conflict in quorum-sensing bacterial populations. Nature 450:411414.

Drescher, K., C. D. Nadell, H. A. Stone, N. S. Wingreen and B. L. Bassler. 2014. Solutions to the public goods dilemma in bacterial biofilms. Curr. Biol. 24:50-55.

Dunne, W. M. 2002. Bacterial adhesion: seen any good biofilms lately? Clin. Microbiol. Rev. 15:155-166.

Fletcher, J. A., and M. Doebeli. 2009. A simple and general explanation for the evolution of altruism. Proc. R. Soc. B Biol. Sci. 276:13-19.

Fudenberg, D., M. A. Nowak, C. Taylor, and L. A. Imhof. 2006. Evolutionary game dynamics in finite populations with strong selection and weak mutation. Theoret. Popul. Biol. 70:352-363.

Garcia, T., L. G. Brunnet, and S. De Monte. 2014. Differential adhesion between moving particles as a mechanism for the evolution of social groups. PLoS Comput. Biol. 10:e1003482.

Garcia, T., and S. De Monte. 2013. Group formation and the evolution of sociality. Evolution 67:131-141.

Garcia, T., G. Doulcier, and S. D. Monte. 2015. The evolution of adhesiveness as a social adaptation. eLife 4:e08595.

Gardner, A., and S. A. West. 2010. Greenbeards. Evolution 64:25-38.

Gillespie, D. T. 2000. The chemical Langevin equation. J. Chem. Phys. 113:297-306.

Guttal, V., and I. D. Couzin. 2010. Social interactions, information use, and the evolution of collective migration. Proc. Natl. Acad. Sci. 107:1617216177.
Hall-Stoodley, L., J. W. Costerton, and P. Stoodley. 2004. Bacterial biofilms: from the natural environment to infectious diseases. Nat. Rev. Microbiol. 2:95-108.

Hamilton, W. D. 1964. The genetical evolution of social behaviour. i. J. Theoret. Biol. 7:1-16.

Hamilton, W. D. 1975. Innate social aptitudes of man: an approach from evolutionary genetics. Biosoc. Anthropol. 133:155.

Hammerschmidt, K., C. J. Rose, B. Kerr, and P. B. Rainey. 2014. Life cycles, fitness decoupling and the evolution of multicellularity. Nature 515:7579.

Hammond, R. A., and R. Axelrod. 2006. Evolution of contingent altruism when cooperation is expensive. Theoret. Popul. Biol. 69:333-338.

Huang, W., C. Hauert, and A. Traulsen. 2015. Stochastic game dynamics under demographic fluctuations. Proc. Natl. Acad. Sci. 112:9064-9069.

Imhof, L. A., D. Fudenberg, and M. A. Nowak. 2005. Evolutionary cycles of cooperation and defection. Proc. Natl. Acad. Sci. 102:10797-10800.

Jansen, V. A. A., and M. van Baalen. 2006. Altruism through beard chromodynamics. Nature 440:663-666.

Joshi, J., I. D. Couzin, S. A. Levin, and V. Guttal. 2017. Mobility can promote the evolution of cooperation via emergent self-assortment dynamics. PLOS Comput. Biol. 13:e1005732.

Keller, L., and K. G. Ross. 1998. Selfish genes: a green beard in the red fire ant. Nature 394:573-575.

Keller, L., and M. G. Surette. 2006. Communication in bacteria: an ecological and evolutionary perspective. Nat. Rev. Microbiol. 4:249-258.

Kogan, O., M. Khasin, B. Meerson, D. Schneider, and C. R. Myers. 2014. Twostrain competition in quasineutral stochastic disease dynamics. Phys. Rev. E 90:042149.

Koschwanez, J. H., K. R. Foster, and A. W. Murray. 2011. Sucrose utilization in budding yeast as a model for the origin of undifferentiated multicellularity. PLoS Biol. 9:e1001122.

Kreft, J. U., and S. Bonhoeffer. 2005. The evolution of groups of cooperating bacteria and the growth rate versus yield trade-off. Microbiology 151:637-641.

Lehmann, L., and L. Keller. 2006. The evolution of cooperation and altruism - a general framework and a classification of models. J. Evol. Biol. 19:1365-1376.

Libby, E., and P. B. Rainey. 2013. A conceptual framework for the evolutionary origins of multicellularity. Phys. Biol. 10:035001.

Lodish, H., A. Berk, S. L. Zipursky, P. Matsudaira, D. Baltimore, J. Darnell. 2000. Mol. Cell Biol. vol. 4. WH Freeman, New York.

McKane, A. J., and T. J. Newman. 2005. Predator-prey cycles from resonant amplification of demographic stochasticity. Phys. Rev. Lett. 94:218102.

Miller, M. B., and B. L. Bassler. 2001. Quorum sensing in bacteria. Ann. Rev. Microbiol. 55:165-199.

Nadell, C. D., J. B. Xavier, and K. R. Foster. 2009. The sociobiology of biofilms. FEMS Microbiol. Rev. 33:206-224.

Nowak, M., and K. Sigmund. 1993. A strategy of win-stay, lose-shift that outperforms tit-for-tat in the prisoner's dilemma game. Nature 364:56.

Nowak, M. A., A. Sasaki, C. Taylor, and D. Fudenberg. 2004. Emergence of cooperation and evolutionary stability in finite populations. Nature 428:646-650.

Pepper, J. W. 2000. Relatedness in trait group models of social evolution. J. Theoret. Biol. 206:355-368.

Powers, S. T., A. S. Penn, and R. A. Watson. 2011. The concurrent evolution of cooperation and the population structures that support it: concurrent evolution of cooperation and population structure. Evolution 65:15271543.

Queller, D. C., E. Ponte, S. Bozzaro, and J. E. Strassmann. 2003. Singlegene greenbeard effects in the social amoeba Dictyostelium discoideum. Science 299:105-106. 
Ratcliff, W. C., R. F. Denison, M. Borrello, and M. Travisano. 2012. Experimental evolution of multicellularity. Proc. Natl. Acad. Sci. . 109:15951600.

Riolo, R. L., M. D. Cohen, and R. Axelrod. 2001. Evolution of cooperation without reciprocity. Nature 414:441-443.

Roberts, G., and T. N. Sherratt. 2002. Behavioural evolution (Communication arising): does similarity breed cooperation? Nature 418: 499-500.

Sahai, E. 2005. Mechanisms of cancer cell invasion. Curr. Opin. Genet. Dev. 15:87-96.

Smith, J. M., and E. Szathmary. 1997. The major transitions in evolution. Oxford Univ. Press, Oxford.

Smukalla, S., M. Caldara, N. Pochet, A. Beauvais, S. Guadagnini, C. Yan, M. D. Vinces, A. Jansen, M. C. Prevost, J. P. Latgé. 2008. Flo1 is a variable green beard gene that drives biofilm-like cooperation in budding yeast. Cell 135:726-737.

Traulsen, A., J. C. Claussen, and C. Hauert. 2006. Coevolutionary dynamics in large, but finite populations. Phys. Rev. E 74:011901.

Traulsen, A., C. Hauert, H. D. Silva, M. A. Nowak, and K. Sigmund. 2009. Exploration dynamics in evolutionary games. Proc. Natl. Acad. Sci. 106:709-712.
Traulsen, A., and M. A. Nowak. 2006. Evolution of cooperation by multilevel selection. Proc. Natl. Acad. Sci. 103:10952-10955.

Traulsen, A., and M. A. Nowak. 2007. Chromodynamics of cooperation in finite populations. PLOS ONE 2:e270.

Traulsen, A., and H. G. Schuster. 2003. Minimal model for tag-based cooperation. Phys. Rev. E 68:046129.

Waters, C. M., and B. L. Bassler. 2005. QUORUM SENSING: cell-to-cell communication in bacteria. Ann. Rev. Cell Dev. Biol. 21:319-346.

West, S. A., A. S. Griffin, and A. Gardner. 2007. Social semantics: altruism, cooperation, mutualism, strong reciprocity and group selection. J. Evol. Biol. 20:415-432.

Xavier, J. B., and K. R. Foster. 2007. Cooperation and conflict in microbial biofilms. Proc. Natl. Acad. Sci. 104:876-881.

Zhang, H., and S. Chen. 2016. Tag-mediated cooperation with nondeterministic genotype-phenotype mapping. EPL 113:28008.

Zukewich, J., V. Kurella, M. Doebeli, and C. Hauert. 2013. Consolidating birth-death and death-birth processes in structured populations. PLoS One 8:e54639.

\section{Supporting Information}

Additional supporting information may be found online in the Supporting Information section at the end of the article.

Table 1. Symbols and parameter values.

Table 2. Symbols and parameter values.

SI-Fig 1. Timeseries with greater noise.

SI-Fig 2. Dynamics in the public goods game.

SI-Fig 3. Simulations using explicit simulations of the system using Gillespie algorithm produces the same qualitative dynamics.

SI-Fig 4. Dynamics with two tags.

SI-Fig 5. Dynamics when strategy and tag mutation rates are different.

SI-Fig 6. Dynamics when solitary cooperators do not suffer the cost of cooperation. 